\title{
Soccer Players Detection Using GDLS Optimization and Spatial Bitwise Operation Filter
}

\author{
Adhi Dharma Wibawa ${ }^{1}$, Atyanta Nika Rumaksari ${ }^{2}$ \\ ${ }^{1}$ Department of Computer Engineering, Institut Teknologi Sepuluh Nopember \\ Jalan Raya ITS, Keputih, Surabaya, Indonesia \\ adhiosa@te.its.ac.id \\ ${ }^{2}$ Universitas Kristen Satya Wacana \\ Jalan Diponegoro No. 52-60, Salatiga, Indonesia \\ atyanta.rumaksari@uksw.edu
}

Received on 07-01-2019, revised on 19-02-2019, accepted on 09-04-2019

\begin{abstract}
The advancement in computer vision technology in order to help coach creates strategy has been affecting the sport industry evolving very fast. Players movement patterns and other important behavioral activities regarding the tactics during playing the game are the most important data obtained in applying computer vision in Sport Industry. The basic technique for extracting those information during the game is player detection. Three fundamental challenges of computer vision in detecting objects are random object's movement, noise and shadow. Background subtraction is an object's detection method that used widely for separating moving object as foreground and non moving object as background. This paper proposed a method for removing shadow and unwanted noise by improving traditional background subtraction technique. First, we employed GDLS (Gradient Decent with Line Search) algorithm to optimize background-foreground separation. Then, we did filter shadows and crumbs-like object pixels by applying digital spatial filter which is created from implementation of digital arithmetic algorithm (bitwise operation). Finally, our experimental result demonstrated that our algorithm outperform conventional background subtraction algorithms. The experiments result proposed method has obtained $80.5 \%$ of F1-score with average 20 objects were detected out of 24 objects.
\end{abstract}

Keywords: background subtraction, object detection, sport science, spatial filter, shadow removal

\section{INTRODUCTION}

A utomatic multiple objects detection in soccer has played an important role in game monitoring system.

Due to high demand of implementing information technology in metric measurement began enormously emerging, research on player's automatic annotation system is flourishing astonishingly. It helps performance analysts and coaches to plan future strategies. Nowadays, this technique has played a crucial role in premiere leagues due to its impact on the competition. The need of sophisticated system which can analyze both players behavioral pattern accurately and their annotation motion during the game can be a valuable competition strategy. Therefore, current game play of international soccer are much more developed than before when such system was not yet implemented. When in each team having the same framework of it, the output will be having same manner. Thus, it will create fierce competition battle between the teams. Furthermore, team without using this strategy will have small change of superiority. Some previous works have the focus on developing automatic detection and tracking algorithm using background subtraction technique [2],[3],[4]. Background subtraction technique is the process based on field image processing technique wherein a foreground is separated from its background in order to further processing. There are two categories of detecting and tracking algorithm based on [1], they were traditional model and recent approach models.

Traditional model deal with the basic characteristic and relatively prone to implement without complex parameter setting. It began from basic model approaches using running average, median and histogram approaches to build background mathematical model [2] [3] [4]. Even though using basic computation model with a focus on an object as random variable which are solved by conventional probabilistic methods, it requires less sources than any other recent model has. However, based on research [1], it can be concluded that, its 
detection quality is lower than the recent model has.

Furthermore, recent models handled challenges such as real-time operation [5], illumination [6], noise cancelation [7], shadow casting [8], and the false alarm [9] in a better way. In other studies, statistical model of background modeling are being modeled from Gaussian distribution [10] [11], support vector model [12], and subspace learning [12] [13]. Implementation of this traditional approaches has been set by the following models such as cluster-based [14], neural networks basis model and estimation-based model [15]. Recent approach works by modeling the object based on subspace approach like (Robust Principle Component Analysis) RPCAapproaches [16], decomposition of sparse matrix [17], subspace tracking based approach [1], low rank minimization [18] [19], robust tensor models [20], outlier detections and transform domain model approach such as FFT, DCT, Walsh, Wavelet, and Hadamard transform approaches [1]. This means, it will add more load in computational effort than any other unification methods.



Fig. 1. Flow of Purposed Methods. Background Subtraction process input image using HSV color map. In foreground detection (2), image difference (3) between background maintenance process (1) will send to find optimum threshold (4), which it employs GDLS algorithm. In Bitwise operation we did concatenate three layers HSV to get filtered binary frame then after output was resulted crumps residue was wiped out by morphological operation.

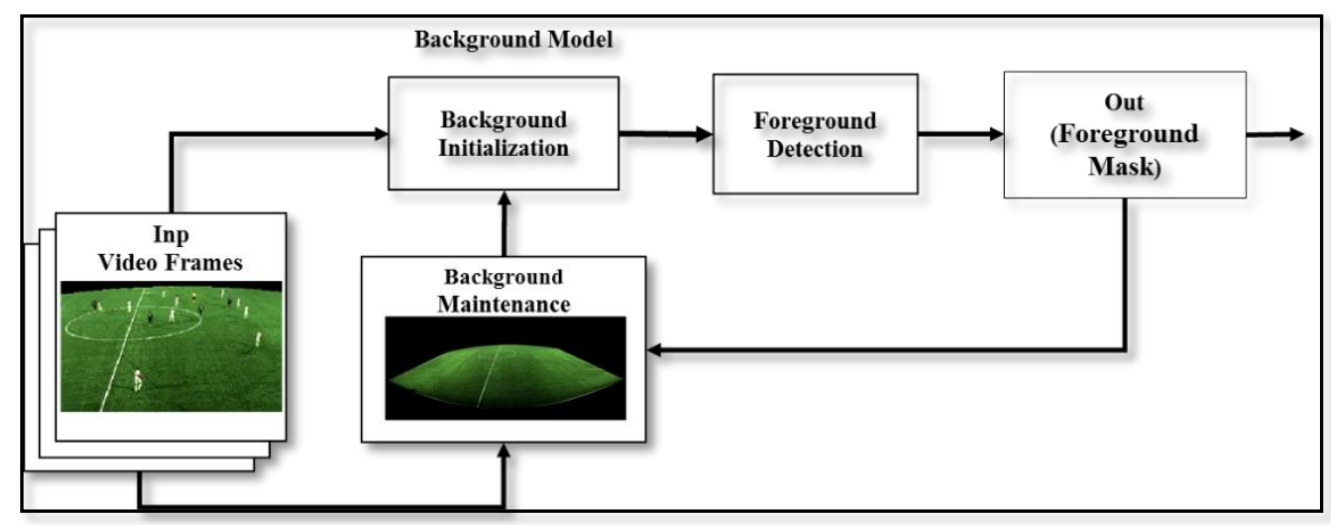

Fig. 2. Background subtraction process. Video Frames as input is responsible to fetch $\mathrm{N}$ frames for maintain the background. Foreground detection is generated by initializing background maintenance process

By observing that two categories, our proposed method is combining above issues, which is using traditional model for less complexity and combining using optimization algorithm and shadow casting technique to achieved higher result like recent model has. We found that binary filter which running in array platform can be used to simplify computational load. We also hypothesize that some other challenges in player's shadow from illumination distribution and noise artefacts from codex or background subtraction error can be solved by spatial bitwise operation filter with an improvement from GDLS algorithm. GDLS or Gradient Decent by Line Search is an algorithm to find the maximum difference between the background and the foreground.

\section{Proposed Method}

The aim of our modified background subtraction method is to find best threshold for binarization and to filter shadow and grumps-like artifact which affect object's brightness level. We grouped our works by 
two categories, the first is background subtraction and the second is spatial filter using bitwise operation for filtering unwanted noise and shadows. Fig 1 shows the flow work of our proposed method

\section{A. Background Subtraction}

In technical definition, background subtraction technique is taking the difference between a background image and object image. Therefore object is separated with its background. In order to separate the background, we need to understand the spatial features on data pixel characteristics [3]. Five features that are commonly used for segmenting or clustering the object are: color, edge, stereo, motion and texture features [1]. Based on our observation in Table 1 the change of illumination (lighting), waking object, sleeping object, and shadows become apparent that those items did not radically change the object background, therefore we hypothesize that using color-based feature for doing object segmentation is fair. However, in soccer which both teams are distinguished by their jersey color, so color-based segmentation technique is used for separating foreground-background.

We found that HSV color has more information about brightness level than RGB color [3]. Therefore, we model an image by $I(u, v)$ where $u$ and $v$ are the pixel coordinate locations and $I$ has three dimension which as $I_{\text {HUE }}, I_{\text {SATURATION }}$, and $I_{V A L U E}$ regarding with three dimensional HSV color-space. The brightness $g($. $)$ is used to level of an object based on point operation. It is given by $I^{\prime}(u, v) \leftarrow g(I(u, v))$. This function affects natural pixel of background $I_{B}$ and foreground $f$. Ideal background subtraction is aiming the best finding of background model $\phi$ which suit with actual image $I_{B}$ or we can say with: $\left\|\phi(u, v)-I_{B}^{\prime}(u, v)\right\| \approx 0$.

From [1], we emphasize that: prior designing suitable methods, we should list key challenge of datasheet features provided that we could best address the key challenges. Table 1 below will help to conduct this activity. Based on theoretical review [1], we found that traditional spatial based operation algorithm is enough, since its datasheet has no dynamic background-foreground changing. Consequently, we choose improved-traditional-background subtraction-based technique as our building block of process method.

Image difference of modeled background and image testing is given by:

$$
f(u, v)=\|\phi(u, v)-I(u, v)\|
$$

In (1), image difference $f(u, v)$ pixels are being thresholdedby $\Gamma=\left[\gamma_{1} \gamma_{2} \gamma_{3}\right]$. In order to get binary masking $G(\Gamma)$, we define $O_{i}$ as detected objects of- $i$. Obtaining higher accuracy of $O_{i}$ leads to higher Fmeasurement. Choosing $\phi(u, v)$ is very crucial, because bad choice of the threshold will lead to false positive detection. Detailed process of background subtraction can be seen at Fig 2. There are four process which are background maintenance, background initialization, foreground detection and masking process.

TABLE I

OBSERVATION OF KEY CHALLENGES

\begin{tabular}{|l|c|}
\hline Challenges & Observation \\
\hline Camera Jitter & No \\
\hline Insufficient quality of camera result of noise & No \\
\hline Camera automatic adjustment & No \\
\hline Time of delay & No \\
\hline Light switch (Illumination changes) & Yes \\
\hline Bootstrapping & No \\
\hline Foreground aperture & No \\
\hline Moved background object & No \\
\hline Inserted background object & No \\
\hline Dynamic background object & No \\
\hline Waking object in the beginning & Yes \\
\hline Sleeping object in the beginning & Yes \\
\hline Shadows & Yes \\
\hline
\end{tabular}

\section{1) Background Initialization}

There is a challenge in traditional background subtracting method where more than half of the required training images are foreground. As a result, the positive background can only be generated partially (half of which is foreground). Therefore, the number of initialization images need to be multiplied until we get 
full background information. Fig 2 showed a causal relationship of background subtraction process. Background maintenance will maintain the change of background initialization, while background initialization will adapt to waking or moving background by taking an average of sampled frames. As a result this will produce an image that contains only the background without foreground

\section{2) Foreground Detection}

In this process, we have done foreground-background separation, by subtracting output background maintenance and input frame. The result is an output image that has close-to-zero value of background pixel and $L$-distance value of object pixel. The aim of this process is extracting foreground from image input $I$ with background model $\emptyset$. Since we need to maintain $f(u, v)$ to be optimum, background maintenance formula is:

$$
\phi_{t+1}(x, y)=(1-\alpha) \phi_{t}(x, y)+\alpha I_{t}(x, y)
$$

Learning rate $\alpha$ is constant in $(0,1)$. Since the background is the static and luminance distribution is constant in each frame. Thus, $\alpha=0$ so that (2) became:

$$
\phi_{t+1}(x, y)=\phi_{t}(x, y)
$$

In addition, we compute background as model approach $\phi *$ for the entire role of image input. Based on our research trial gathering $\mathrm{N}>50$ frames for calculating $\phi$ is enough, because stationary cameras with constant illumination were being placed in the field.

$$
\phi *_{t}(x, y)=\frac{1}{N} \sum_{i=1}^{N} I_{i}(x, y)
$$

Algorithm 1: threshold reinforcement using GDLS

$$
\begin{aligned}
& \hline \text { Input }: \text { Video Sequences }\left\{f_{t}(\Gamma)\right\} \\
& \text { Video Sequences }\left\{\psi_{t}(\Gamma)\right\} \\
& \text { initial } G \Gamma_{\text {Step } t}=\operatorname{rand}(.) \\
& t=1, \ldots \ldots . ., N . d
\end{aligned}
$$

Output :arg min $\delta$.

Initial $y_{1}$ for $1: \mathrm{T}_{1}$,

$\gamma_{2}$ for $1: \mathrm{T}_{2}$,

$\gamma_{3}$ for $1: \mathrm{T}_{3}$,

Finding a step size $\eta_{t}$ such that:

$$
G(\Gamma)=\|f(\Gamma)-\psi(\Gamma)\|<G \Gamma_{\text {step }}
$$

Store variable to:

$$
\left(\delta \rightarrow \delta_{t}\right) \leftarrow-\eta_{t} \nabla_{\Gamma(t-1)}-\psi(\Gamma)
$$

Update $\delta_{t} \Gamma_{t} \leftarrow \Gamma_{t-1}+\delta_{t}$.

$$
\text { End of } \gamma_{3}
$$

End of $\gamma_{2}$

End of $\gamma_{1}$ 


\section{3) Foreground Masking}

The proposed method is using optimization method Gradient Decent with Line Search algorithm $(G D L S)$. Our problem statement is to find $\arg \min _{\Gamma} f(\Gamma)$. Possibly subject to constant $\Gamma$.

Where finding $\min f(\Gamma)$ is equal to find best formation of $\Gamma=\left[\gamma_{1} \gamma_{2} \gamma_{3}\right]$ and $\Gamma \in \mathbb{R}^{n} . f(\Gamma) \rightarrow$ $\|g(\Gamma)-\psi(\Gamma)\|$ with $\varphi(\Gamma)=g(\Gamma)+f(\Gamma)$. We can say that $f(\Gamma)$ is the residue from equation $\psi(\Gamma)$. In this experiment, we define image $\psi$ as ground truth

From calculus, we recall that minimum of $f(\Gamma)$ must lie at point when $\frac{\delta f(\Gamma)}{d(\Gamma)}=0$ and it will automatically maximize $p(g \mid f)$. We iterate and sort it based on the number of dimension. Each optimal value is found using GDLS. For each dimension step size $\eta_{t}$ for each $t$ time, will produce variable $\delta$. This variable will be updated for future comparison $\operatorname{in} G(\Gamma)<G\left(\Gamma_{\text {step }}\right)$. Note that whenever the weight of previous $-\eta_{t} \nabla_{\Gamma(t-1)} \approx\|f(\Gamma)-\psi(\Gamma)\|$ are specified, we have a complete rule of future comparison. This means that the difference value between ground truth and foregorund is clearly separated.

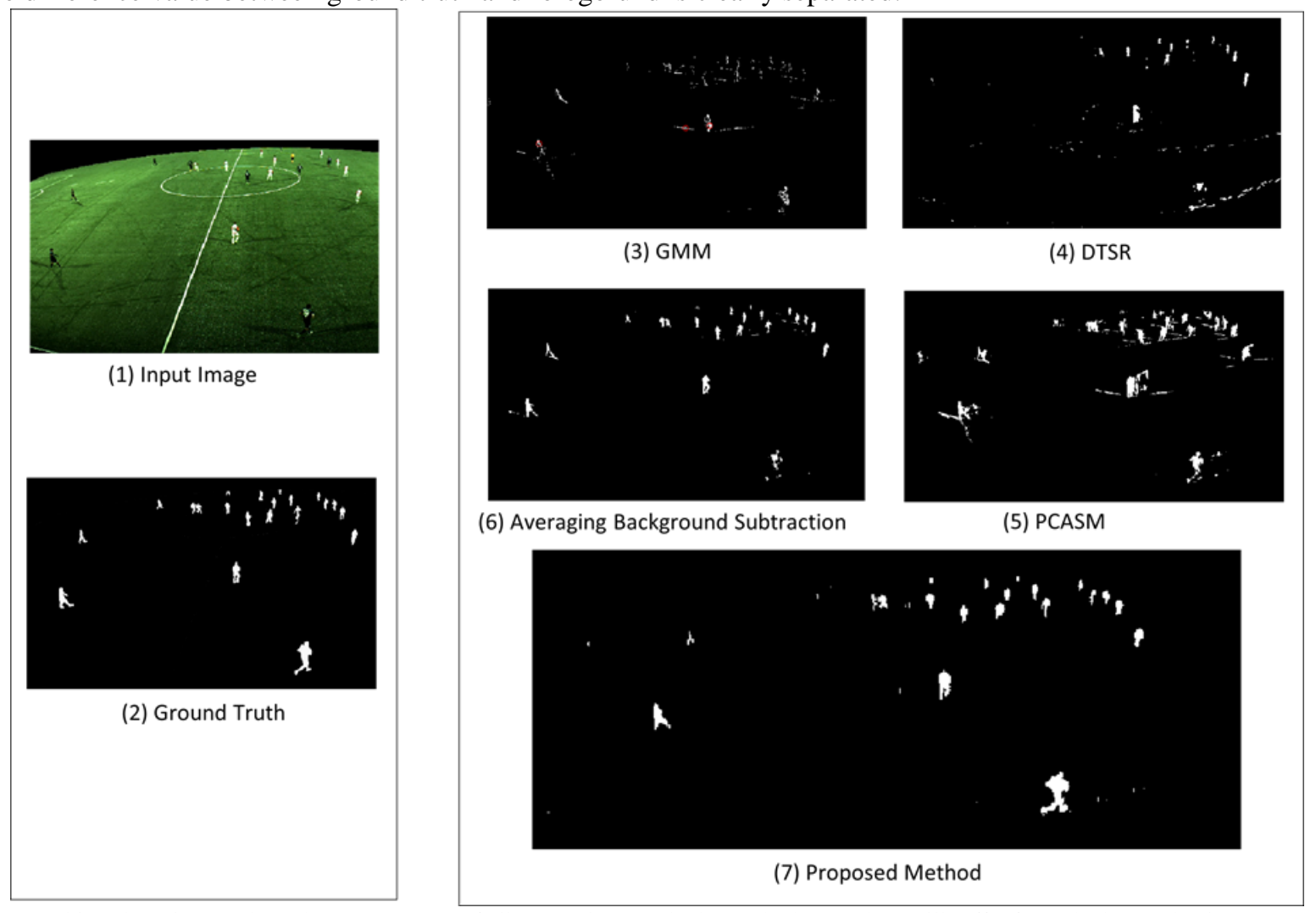

Fig. 3. Object detection result on the test sequence of all comparison methods. Method (3) and (5) still suffer from shadow and noise present, while (4) and (6) able to cast the noise and shadows but still suffer from uncomplete figure

Thus, when all the iteration is done, we can sort based on min $\delta$ which it corresponds to $\arg \min _{\Gamma} f(\Gamma)$. Algorithm 1 summarizes the rules-learning algorithm. 




Fig 4. Bitwise Filter Logical gate diagram

\section{B. Spatial Filter using Bitwise Operation}

Bitwise operator is found based on spatial information form $f(\Gamma)$. Since this is three color represented in HSV color format, then finding best threshold should be observed by these color. Saturation is capturing distance between origin and Hue value. On the other hand, saturation is the radius between origin and hue point. Hue is the angle around the central vertical axis. Value is the height of cylindricalcoordinate from the origin [21].

Output $\mathrm{H}, \mathrm{S}$ and V were derived from by applying threshold $\Gamma$ to $f(\Gamma)$. The spatial filter does not need progressive computation because it was applying the array-to-array computation. Progressiveness in computation will lead to more memory allocation, consequently, it result in higher complexity than direct computation. Fig 4 showed how we formed a filter. It began with a AND function of saturation color (b) and value (c) color component to filter the shadows (d) in detected foreground. Because it has incomplete form of object's form, another additional operation is needed for recovering lost component by using Hue color (a). Thus, OR operation between (d) and (a) were resulted. This operation gets full figure of an object but suffering from line fields that were detected as foreground (e). Hence for the final step, we do AND operation between the previous result (e) with value component (c) again to get final output (f). Fig 4 presents detailed operations as truth table of filter's logic gate.

\section{Dataset}

This proposed method was compared with four widely used technique PCA based and spatial smoothing model (PCASM) encourage by [9], Gaussian Mixture Model (GMM) [11] [8], Dynamic thresholding for shadow removal Algorithm (DTSR) [22] and Point Operation Background Subtraction (POBS). All object detector are picked based on [1] recommendation in overcoming key challenges.

Typical datasets are consist of 26,222 frames of 40 minutes video h264 format provided by [23]. Each frame wide 2000 by 4500 pixels. The datasheet has some characteristics which can be our challenge, are summarized bellow:

- $\quad$ Skewed lighting distribution, sees Fig. 5 (left).

- Dynamic random foreground movement.

- Shadow is detected as foreground.

- Since the video is constructed by the panoramic synthetic video camera, then object's shape is changing in curved area.

- Occlusion is occurred and

- The object will appear intermittent because it was located in distance. 


\section{Evaluation Matrix}

Our experiments were using standard metric for the testing performance of object detection algorithm [24]. The quantitative comparison is performed using F1-score measurement which developed by True Positive (TP), False Negative (FN), and False Positive (FP). Where $X$ as an input image and $\Omega$ as ground truth image.

$$
\mathrm{F}_{1}=\frac{2 \cdot \mathrm{TP}}{2 \cdot \mathrm{TP}+\mathrm{FN}+\mathrm{FP}}
$$



Fig. 5. Left: skewed lighting distribution from court side to middle field. Right: jersey player most likely equal with the darkest background

\section{RESULTS AND DISCUSSION}

We explained about technical flow of building proposed method in chapter II previously. In this session we will discuss the result by implementing given methods. Fig 1 (2) shows the image difference result. Based on the experiment, we acquire that all channels have their own characteristics. In image difference $d H$, it preserves grayscale value greater than $20 \%$ of lower parts, therefore we can see the field line and bright jersey have more dominant than the field. While, $d S$ preserves greater field line value and some parts of dark jersey players. We also got $d V$ preserves higher amount of total players value and their shadows. Thus, we could combine threshold for $[d H, d S, d V]$ to produce a better form of foreground detector. However, some testing regarding the algorithm and the result need to be done to measure the effectieveness in applying new threshold method. Critical threshold value is also need to be determined by further experiment.

We proposed GDLS optimization technique for finding the threshold. We performed training process in all the training sequences, and as a result, we achieved threshold $\Gamma$ with minimum $\delta$. Based on our research using Algorithm 1 approaches, we got $\Gamma=\left[\begin{array}{ll}0.1 & 0.5 \\ 0.1\end{array}\right]$. In each 30 frames processing, we found this threshold after total 6,480 times of iteration. The bitwise operation will filter thresholded output. All described stages of each logic gate's result can be found in Fig 4. General binary equation of bitwise operation filter is: $A N D(\boldsymbol{V}, O R(A N D(\boldsymbol{S}, \boldsymbol{V}), \boldsymbol{H}))$. Fig 4(f) shows that the shadows can be filtered effectively by given filter.

However, there were numbers of crumbs-like artifacts that sometimes occur (Fig 3 (7)) which considered as noise. Even tough there were still many noise artifacts which lead to false positive detection, it did not affect the whole detections. F1-score in Table II showed that our result was 0.805 superior to other existing algorithms. TP, FN, and FP were $0.731,0.1754$ and 0.1793 respectively. For benchmarking our result, we compared against GMM, DTSR, PCASM, and POBS and they were resulting F1 score 0.281, 0.456, 0.648 and 0.754 respectively. 


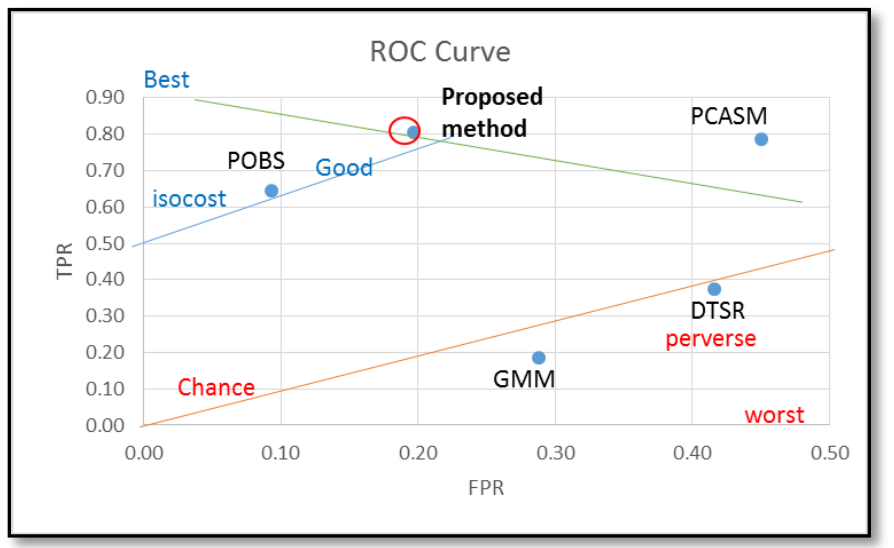

Fig. 6. Illustration of ROC curve, proposed method lies inside diagonal isocost line, which representing good equal cost-performance [25]

TABLE II

PLAYERS DETECTION ON VARIOUS METHODS

\begin{tabular}{|c|c|c|c|c|}
\hline Methods & TP & FN & FP & F1 \\
\hline GMM [11] [8] & 0.1679 & 0.7385 & 0.068 & 0.281 \\
\hline DTSR [22] & 0.339 & 0.5675 & 0.2419 & 0.456 \\
\hline PCASM [9] & 0.713 & 0.1934 & 0.5837 & 0.648 \\
\hline POBS & 0.5856 & 0.3209 & 0.0602 & 0.754 \\
\hline Proposed Method & $\mathbf{0 . 7 3 1}$ & $\mathbf{0 . 1 7 5 4}$ & $\mathbf{0 . 1 7 9 3}$ & $\mathbf{0 . 8 0 5}$ \\
\hline
\end{tabular}

Skewed lighting distribution of field will result gradual change of brightness from bright to dark and it was affecting object's pixel value (Fig 7-left) to become more likely close to pixel's field. Those objects were hard to distinguish and it will lead detector produce miss detection. Fig 7-middle showed our error detection coded object as background. Fig 7-right showed recovery process of suffered object processed by morphological operation. Another similar illumination challenge was shown in Fig 7 (left) and our proposed method were successfully subtracted background into foreground. In other word, traditional background subtraction by using equation-(4) was proven successfully for separating background from dynamic foreground. Dataset were using camera's panoramic format [23], therefore objects will change in shape, when they were locating on curved frame. This phenomenon can be seen in Fig 3. This condition will affect image representation, so that object location will be differ than it should be. Consequently for accurately determine the object, coordinate points required coefficients of camera curvature constraint.
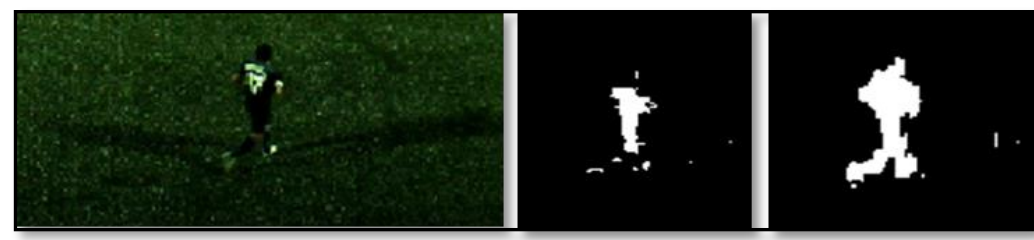

Fig.7. Left: image source, middle: skewed distribution of light causing detector unable to detect some body parts, right: object recovery using morphology operation

Grumps-like artifacts noise appear because there were error in video coding format or some moving pixels which coded as foreground. It can be seen at Fig 7 (middle). By employing median spatial filter, these noises can be eradicated.

Proposed method was locating in AUC (Area Under Curve) (Fig 6) which is an area under the trapezoid curve between the blue and green line. From that curve, proposed method has been classified as good acceptable method [25]. Therefore, we can conclude that in Table II proposed method is superior to other methods is justifiable. In Fig 3 we can see that there were two methods, which stated as the acceptable method they were POBS $75.4 \%$ and proposed method, whereas PCASM was in barely acceptable in $64.8 \%$ 
and other methods were on unacceptable perverse line, GMM and DTSR both less than 50\% F1 measure.

\section{CONCLUSION}

We found that our new method of combining traditional background subtraction [1] with thresholding optimization and a bitwise binary spatial filter is evident to overcome noises and object's shadow and also could handle several challenges as skewed field lighting distribution and dynamic foreground motion. Eventhough we could not get the maximum result by detecting all players, however, we obtained F1-score as 0.805 outperformed other methods, as we can see in Table II. From AUC diagram in Fig 6, proposed method was also showed superior because it was located inside in acceptable region.There are two remaining challenges that need to be handled in the future, i.e. intermittent of object's occurrences and occlusion. These problems are still happen in our condition. However, there are possibilities to handle it by combining object detection with tracking methods by calculating future estimated position. Moreover, for foreground detection, a better threshold need to be found by further experiment so that the result could be optimum regarding preserving the grayscale in the image.

\section{REFERENCES}

.[1] T. Bouwmans, "Traditional and recent approaches in background modeling for foreground detection: An overview," Computer Science Review, Vols. 11-12, pp. 31-66, May 2014.

[2] Z. Yi and F. Liangzhong, "Moving object detection based on running average background and temporal difference," in 2010 IEEE International Conference on Intelligent Systems and Knowledge Engineering, Hangzhou, 2010.

[3] P. Perez, C. Hue, J. Vermaak and M. Gangnet, "Color-Based Probabilistic Tracking," in ECCV 2002, LNCS 2350, Berlin Heidelberg, 2002.

[4] Z. Zeng, J. Jia, D. Yu, Y. Chen and Z. Z. Zhu, "Pixel modeling using histograms based on fuzzy partitions for dynamic background subtraction," IEEE Transactions on Fuzzy Systems, vol. PP, no. 99, pp. 1 - 1, 2016.

[5] C. Hsiang-Chieh, C. Ya-Ching, L. Nai-Jen, W. Cheng-Feng and W. Wen-June, "Real-Time People Counting Method with Surveillance Cameras Implemented on Embedded System," in Proceedings of the World Congress on Engineering and Computer Science 2013 Vol I, San Fransisco, USA, 2013.

[6] S. T, "A Moving Object Segmentation Method for Low Illumination Night Videos," in Proceedings of the World Congress on Engineering and Computer Science 2008, San Fransisco, USA, 2008.

[7] F. Atena, S. Abdolhossein and S. Jamshid, "Document Image Noises and Removal Methods," in Proceedings of the International MultiConference of Engineers and Computer Scientists 2013 Vol I, Hong Kong, 2013.

[8] P. Kaewtrakulpong and R. Bowden, "An Improved Adaptive Background Mixture Model for Real-time Tracking with Shadow Detection," in Video-Based Surveillance Systems, Springer US, 2002, pp. 135-144.

[9] X. Gengjian, L. Song, J. Sun and J. Zhou, "Foreground detection: Combining background subspace learning with object smoothing model," in 2013 IEEE International Conference on Multimedia and Expo (ICME), San Jose, CA, 2013.

[10] A. M. Rahman, B. Ahmed, A. M. Hossian and N. I. Mondal, "An adaptive background modeling based on modified running Gaussian average method," in 2017 International Conference on Electrical, Computer and Communication Engineering (ECCE), Bangladesh, 2017.

[11] L. Yao and L. Miaogen, "An Improved Mixture-of-Gaussians Background Model with Frame Difference and Blob Tracking in Video Stream," The Scientific World Journal, vol. 2014, p. 9 pages, 2014.

[12] T. Bouwmans, "Recent Advanced Statistical Background Modeling for Foreground Detection - A systematic Survey," Recent Patents on Computer Science, pp. 147-176, 2011.

[13] Z. Zhou and Z. Jin, "Two-dimension principal component analysis-based motion detection framework with subspace update of background," IET Computer Vision, vol. 10, no. 6, pp. 603-612, $10 ` 6$.

[14] Y. Zhang, Q. He, H. Wang, G. Guan, T. Xu and H. Chen, "Background subtraction based on pixel clustering," in 2016 IEEE International Conference on Information and Automation (ICIA), Zhejiang, 2016.

[15] S. Sumpeno, M. Hariadi and T. Aoki, "A Region-based Approach using LVQ for Semi-Automatic Video Object Extraction Technique," in 8th Seminar on Intelligent Technology and Its Applications, Surabaya, Indonesia, 9-10 May 2007.

[16] C. Guyon, T. Bouwmans and E.-h. Zahzah, "Robust Principal Component Analysis for Background Subtraction: Systematic Evaluation and Comparation Analysis," in INTECH, Principal Component Analysis, Book 1, In Tech, March 2012, pp. $223-238$.

[17] S. E. Ebadi, V. G. Ones and E. Izquierdo, "Efficient background subtraction with low-rank and sparse matrix decomposition," in 2015 IEEE International Conference on Image Processing (ICIP), Quebec City, 2015.

[18] X. Zhou, C. Yang and W. Yu, "Moving object detection by detecting contiguous outliers in the low-rank representation," in IEEE Trans. Pattern Anal. 35, 2013.

[19] L. Xiong, X. Chen and S. J., "Direct robust matrix factorization for anomaly detection," in International Conference on Data Mining, ICDM 2011, 2011.

[20] A. Cichocki, A. Phan, R. Zdunek and S. Amari, "Nonnegative Matrix and Tensor Factorization Applications to Exploratory MultiWay Data Analysis and Blind Source Separation," in Willey, 2009.

[21] R. H. Hunt and M. Pointer, Measuring Color, 4th Edition, Wiley, 2011.

[22] M. Taha, H. H. Zayed, M. E. Khalifa and T. Nazmy, "Moving Shadow Removal for Multi-Objects Tracking in Outdoor Environments," International Jurnal of Computer Applications, vol. 97, no. 10, pp. 43-51, July 2014. 
[23] S. Arne Pettersen, D. Johansen, H. Johansen, V. Berg Johansen, V. Reddy Gaddam, A. Mortensen, R. Langseth, C. Friwodz, H Kvale Stensland and P. Halvorsen, "Soccer Video and Player Position Dataset," in Singapore ACM 19-21 March 2014, Singapore, 2014.

[24] F. Ma and S. Nong, "Background Subtraction Based on Multi-Channel SILTP," in Computer Vision - ACCV 2012 Workshops, vol. 7728, Berlin, Springer, 2013, pp. 73-84.

[25] M. W. D. Powers, "Evaluation: From Precision, Recall, and F-Factor to ROC, Informedness, Markedness and Correlation," International Cognitive Science Conference, 2003.

[26] T. T. Zin, P. Tin, T. Takashi and H. Hiromitsu, "A Novel Probabilistic Video Analysis for Stationary Object Detection in Video Survillance Systems," IAENG International Journal of Computer Science, vol. 39, no. 3, pp. 295-306, 2012. 